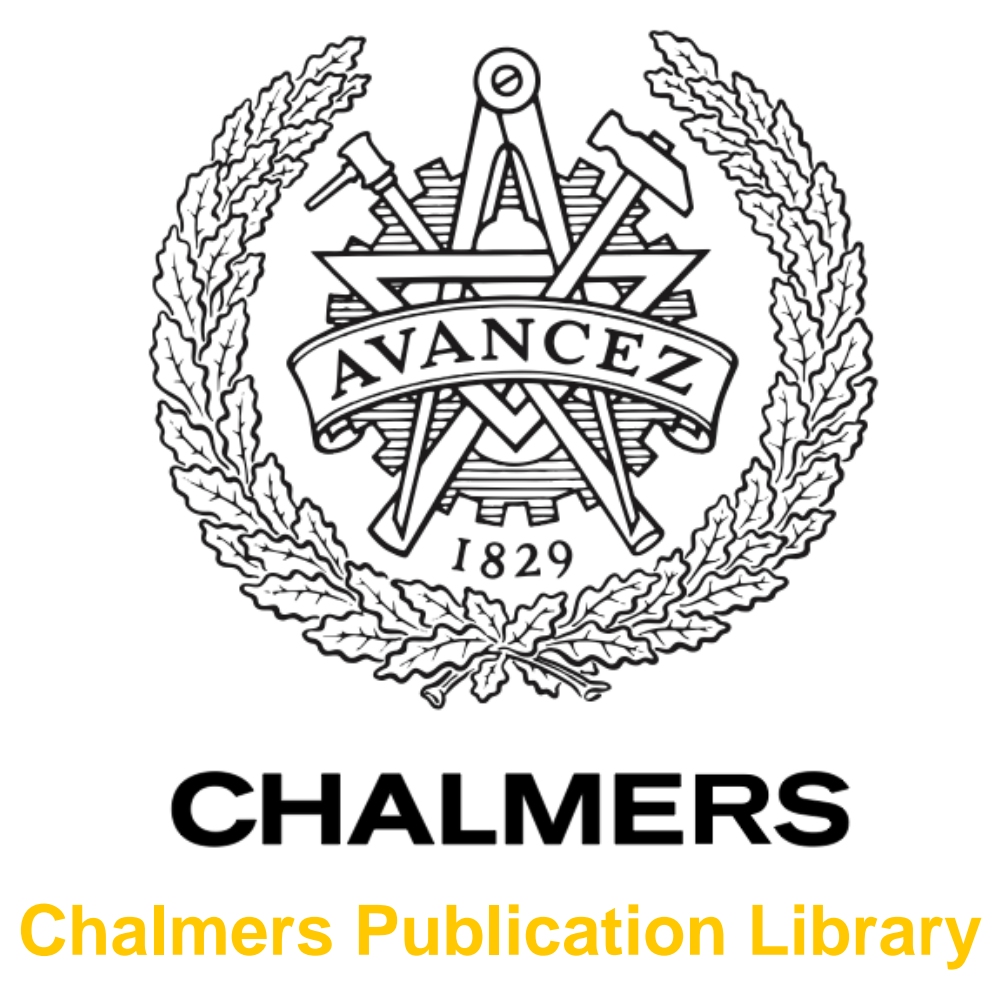

\author{
Eigenbeam transmission over line-of-sight MIMO channels for fixed microwave \\ links
}

This document has been downloaded from Chalmers Publication Library (CPL). It is the author's version of a work that was accepted for publication in:

Proceedings - ITG/IEEE Workshop on Smart Antennas (WSA)

\author{
Citation for the published paper: \\ Mecklenbräuker, C. ; Matthaiou, M. ; Viberg, M. (2011) "Eigenbeam transmission over line- \\ of-sight MIMO channels for fixed microwave links". Proceedings - ITG/IEEE Workshop on \\ Smart Antennas (WSA)
}

http://dx.doi.org/10.1109/WSA.2011.5741915

Downloaded from: http://publications.lib.chalmers.se/publication/136015

Notice: Changes introduced as a result of publishing processes such as copy-editing and formatting may not be reflected in this document. For a definitive version of this work, please refer to the published source. Please note that access to the published version might require a subscription.

Chalmers Publication Library (CPL) offers the possibility of retrieving research publications produced at Chalmers University of Technology. It covers all types of publications: articles, dissertations, licentiate theses, masters theses, conference papers, reports etc. Since 2006 it is the official tool for Chalmers official publication statistics. To ensure that Chalmers research results are disseminated as widely as possible, an Open Access Policy has been adopted.

The CPL service is administrated and maintained by Chalmers Library. 


\title{
Eigenbeam Transmission over Line-of-Sight MIMO Channels for Fixed Microwave Links
}

\author{
Christoph F. Mecklenbräuker*, Michail Matthaiou ${ }^{\dagger}$, and Mats Viberg ${ }^{\dagger}$ \\ * Christian Doppler Laboratory for Wireless technologies for sustainable mobility \\ Institute of Telecommunications, Vienna University of Technology, Vienna, Austria \\ $\dagger$ Department of Signals and Systems, Chalmers University of Technology, Gothenburg, Sweden \\ Email: cfm@nt.tuwien.ac.at, \{michail.matthaiou, viberg\}@chalmers.se
}

\section{ABSTRACT}

We propose and analyze eigenbeam transmission over lineof-sight (LOS) multiple-input multiple-output (MIMO) channels with linear reception. In particular, we consider fixed point-to-point microwave links for which the singular value decomposition of the LOS MIMO channel matrix is analytically derived. We demonstrate that the Tx eigenbeams can be defined without channel state information at the transmitter and that the beams admit an insightful physical interpretation. We also evaluate the performance of a lattice-reduction aided linear receiver through numerical simulations.

\section{INTRODUCTION}

The widespread deployment of Third Generation Long Term Evolution (LTE) networks based on MIMO-OFDM technology will increase the demand for high-reliability high-rate fixed microwave links for implementing the backhaul links. In fact, the sum-rate of an LTE base station is anticipated to exceed $1 \mathrm{Gbit} / \mathrm{s}$, which makes MIMO technology a strong candidate to realize such high data rates. In typical microwaves links, the presence of a strong LOS component is highly likely and hence the fading statistics can be efficiently modeled via the Ricean distribution with a rather high $K$-factor. On this basis, the area of Ricean fading MIMO channels has recently attracted considerable research interest (see [1]-[3] and references therein among others).

Tulino et al. have found the optimum input covariance matrix assuming statistical channel state information at the transmitter (CSIT), i.e. statistical waterfilling, [4] for both Rayleigh and Ricean channels. Riegler and Taricco have evaluated the ergodic capacity of the asymptotic separately-correlated Ricean fading MIMO channel with interference [5], [6]. Nabar et al. have defined a critical data rate for Ricean MIMO fading channels [7] below which the MIMO channel behaves like an additive white Gaussian noise (AWGN) channel. Bøhagen et $a l$. have analytically evaluated the ergodic capacity of dualbranch Ricean MIMO systems, where the minimum number of antennas is equal to two [1]. The statistics of the signal to noise ratio (SNR) of each eigenmode for spatial multiplexing MIMO systems can be found in [2]. This is accomplished by deriving analytical expressions for the marginal eigenvalues of the instantaneous correlation matrix. A similar analysis for the case of optimized LOS MIMO configurations was recently performed by Matthaiou et al. in [3], using tools from random matrix theory. These optimized configurations, originally proposed in [8], [9], are of high practical importance since they lead to subchannel orthogonality which is a key condition for capacity maximization. The key idea is to place the antenna elements sufficiently far apart so that the spatial LoS responses become unique with a phase difference of $\pi / 2$. The main disadvantage of these topologies though is that the optimum spacing can sometimes be infeasible due to space limitations (e.g. mobile handsets). Hence, an alternative solution for achieving subchannel orthogonality with practical inter-element spacings is highly desirable.

In this paper, we elaborate on the performance of fixed point-to-point microwave links with a strong LOS component by considering the case of eigenbeam transmission over MIMO eigenmodes. In particular, the main paper contributions can be summarized as follows:

- We first consider symmetric $2 \times 2$ MIMO systems, for which the eigedecomposition and singular value decomposition (SVD) of the channel matrix are derived in a tractable closed-form, which offers very useful insights into the eigenbeam design.

- Our analysis is then extended to $4 \times 4$ MIMO systems which reveals an interesting Kronecker relationship with the $2 \times 2$ case. This dependency holds not only for the SVDs but also for the actual channel matrices.

- The performance of the considered systems is evaluated using three different receivers. Our numerical results indicate that a significant performance gain can be achieved by implementing a lattice-reduction aided Zero-forcing (ZF) scheme. Further, these gains are achievable even for ill-conditioned channel matrices which correspond to practical values of inter-element spacing (i.e. much smaller than the optimum spacings proposed in [8], [9]).

The remainder of the paper is structured as follows: In Section II the channel model used throughout the paper is presented. In Section III, we present the eigenbeam design for $2 \times 2$ systems while Section IV is devoted to $4 \times 4$ systems. The concept of eigenbeam transmission using lattice-reduction $\mathrm{ZF}$ is discussed in Section V. A performance evaluation is given in Section VI while the key conclusions of the paper are summarized in Section VII. 
Notation: We use upper and lower case boldface to denote matrices and vectors, respectively. The $(m, n)$-th entry of an $M \times N$ matrix $\mathbf{X}$ is $\mathbf{X}_{m n}$ with $1 \leq m \leq M$ and $1 \leq n \leq$ $N$. The symbols $(\bullet)^{T}, \otimes$ represent the matrix transpose and Kronecker product, respectively.

\section{LOS MIMO CHANNEL MODEL}

The considered channel model includes a LOS component, a frequency-selective ground reflection, and very limited multipath otherwise [10]-[13]. The fading statistics of such channels typically follow the Ricean distribution where the Ricean $K$-factor depends on the rain intensity [10], [14]. The channel matrix, $\boldsymbol{H}(t)$ is modeled according to [13]

$$
\boldsymbol{H}(t)=\sqrt{\frac{K}{K+1}} \boldsymbol{H}_{0}+\sqrt{\frac{1}{K+1}} \boldsymbol{H}_{\mathrm{rain}}+\varrho \mathrm{e}^{-j \omega \tau} \boldsymbol{H}_{\mathrm{GR}}(t)
$$

where $\boldsymbol{H}_{0}$ is the deterministic LOS component while $\boldsymbol{H}_{\mathrm{GR}}$ is the ground reflection matrix. The ground reflection coefficient $\varrho$ and ground reflection delay $\tau$ are fixed and constant for all sub-channels. More specifically, we assume that $\boldsymbol{H}_{\mathrm{GR}}(t)$ is a zero mean circularly complex Gaussian random matrix whose elements have covariances defined by the Kronecker MIMO channel model. We simplify this model by neglecting the delay of the ground reflection. In other words, we adopt (1) with $\tau=0$ and

$$
\boldsymbol{H}_{\mathrm{GR}}(t)=\boldsymbol{R}^{1 / 2} \boldsymbol{W}(t) \boldsymbol{T}^{1 / 2} .
$$

The Hermitian positive definite matrices $\boldsymbol{R}$ and $\boldsymbol{T}$ characterize the spatial receive and transmit covariance, respectively. The random matrix $\boldsymbol{W}(t)$ has i.i.d. zero mean circularly complex Gaussian random elements (Rayleigh fading). The matrices $\boldsymbol{R}$ and $\boldsymbol{T}$ are calculated from the power distribution of the ground reflection versus elevation angle $\vartheta$ by assuming uncorrelated scattering [15, Eqs. (23) and (24)]. A detailed derivation is provided in the Appendix.

\section{III. $2 \times 2$ FREQUENCY-FLAT LOS-MIMO MODEL}

We assume a highly symmetric $2 \times 2$ antenna geometry as depicted in Fig. 1 which represents a typical backhaul link.

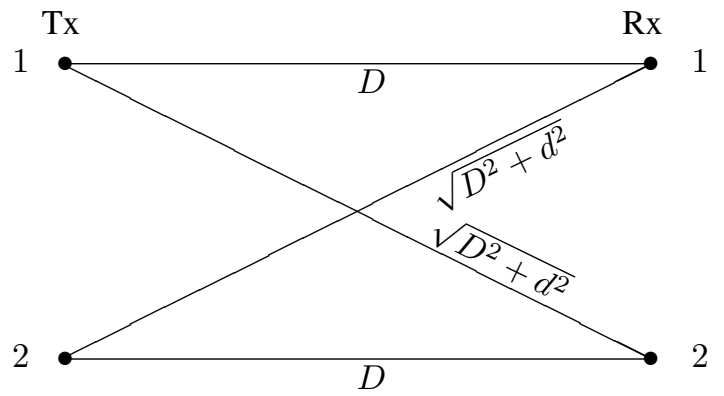

Fig. 1. Highly symmetric $2 \times 2$ LOS MIMO geometry. Typical parameters are $D=5 \mathrm{~km}, 1 \mathrm{~m}<d<5 \mathrm{~m}, f=32 \mathrm{GHz}$ [12, Fig. 3.3, po. 28].

In this case, the distances between all pairs of transmit and receive antenna elements can be worked out via simple geometrical tools as follows

$$
\begin{aligned}
& d_{11}=d_{22}=D \\
& d_{12}=d_{21}=\sqrt{D^{2}+d^{2}} \approx D+\frac{d^{2}}{2 D} .
\end{aligned}
$$

The input-output relationship in free-space can be expressed according to

$$
\left(\begin{array}{l}
r_{1} \\
r_{2}
\end{array}\right)=\left(\begin{array}{ll}
\mathrm{e}^{-j k d_{11}} & \mathrm{e}^{-j k d_{12}} \\
\mathrm{e}^{-j k d_{21}} & \mathrm{e}^{-j k d_{22}}
\end{array}\right)\left(\begin{array}{c}
s_{1} \\
s_{2}
\end{array}\right)+\left(\begin{array}{c}
n_{1} \\
n_{2}
\end{array}\right) .
$$

where $s_{k}, r_{k}, k=\{1,2\}$ correspond to the transmitted and received symbols, respectively while $n_{k}, k=\{1,2\}$ denotes the AWGN term. This results in the following idealized LOS channel matrix

$$
\boldsymbol{H}_{0}=\mathrm{e}^{-j k D}\left(\begin{array}{cc}
1 & a \\
a & 1
\end{array}\right)
$$

where $k=\frac{2 \pi}{\lambda}$ is the wavenumber corresponding to the carrier wavelength $\lambda$, while

$$
a=\mathrm{e}^{-j k D\left(\sqrt{1+(d / D)^{2}}-1\right)} \approx \mathrm{e}^{-j \frac{k d^{2}}{2 D}} .
$$

Note that $\boldsymbol{H}_{0}$ is a square complex matrix which commutes with its conjugate transpose. This ensures that $\boldsymbol{H}_{0}$ is convertible to diagonal form by a unitary transform. The eigendecomposition of $\boldsymbol{H}_{0}$ reads

$$
\begin{aligned}
& \boldsymbol{\Lambda}_{0}=\mathrm{e}^{-j k D}\left(\begin{array}{cc}
1+a & 0 \\
0 & 1-a
\end{array}\right) \\
& \boldsymbol{V}_{0}=\frac{1}{\sqrt{2}}\left(\begin{array}{cc}
1 & -1 \\
1 & 1
\end{array}\right)=\left(\begin{array}{ll}
\boldsymbol{v}_{0}^{+} & \boldsymbol{v}_{0}^{-}
\end{array}\right)
\end{aligned}
$$

and $\boldsymbol{H}_{0}=\boldsymbol{V}_{0} \boldsymbol{\Lambda}_{0} \boldsymbol{V}_{0}^{T}$. We note that the modal matrix $\boldsymbol{V}_{0}$ is real-valued and orthogonal. In fact, $\sqrt{2} \boldsymbol{V}_{0}$ is the Hadamard matrix of order 2. Most importantly, the modal matrix $\boldsymbol{V}_{0}$ does not depend on model parameters.

The SVD of $\boldsymbol{H}_{0}=\boldsymbol{U}_{0} \boldsymbol{S}_{0} \boldsymbol{V}_{0}^{H}$ is closely related to the eigendecomposition. More specifically, using (6) and (7), we can get

$$
\begin{aligned}
\boldsymbol{U}_{0} & =\left(\begin{array}{cc}
\zeta_{0}^{+} \boldsymbol{v}_{0}^{+} & \zeta_{0}^{-} \boldsymbol{v}_{0}^{-}
\end{array}\right) \\
\boldsymbol{S}_{0} & =\left(\begin{array}{cc}
|1+a| & 0 \\
0 & |1-a|
\end{array}\right)
\end{aligned}
$$

with the phase rotations $\zeta_{0}^{+}, \zeta_{0}^{-}$being given in closed-form according to

$$
\begin{aligned}
\zeta_{0}^{+} & =\frac{1+a}{|1+a|} \mathrm{e}^{-j k D} \\
\zeta_{0}^{-} & =\frac{1-a}{|1-a|} \mathrm{e}^{-j k D} .
\end{aligned}
$$

The chosen association of the phase rotations with the $\boldsymbol{U}_{0}$ matrix is not at all mandatory. We exploit the non-uniqueness of the SVD and move the phase rotations from the left factor $\boldsymbol{U}_{0}$ to the right factor $\boldsymbol{V}_{0}^{H}$. With this definition of the SVD, we can use the transmit eigenbeams without requiring channel state information at the transmitter (CSIT). Practically, the model (5) is valid only approximately due to multipath propagation effects (bottom bounce, etc.). 


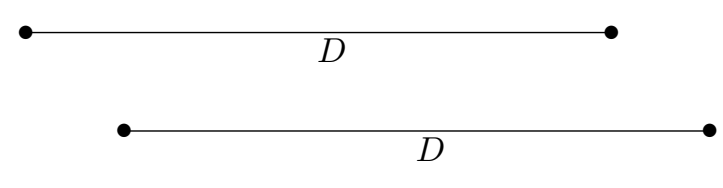

Tx

$\mathrm{Rx}$

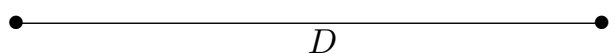

Fig. 2. Highly symmetric $4 \times 4$ geometry using square arrays on both sides of the MIMO link.

\section{IV. $4 \times 4$ FREQUENCY-FLAT LOS-MIMO MODEL}

Let us now extend the analysis of Section III to the case of $4 \times 4$ configurations. For this reason, we consider the highly symmetric $4 \times 4$ square array topology shown in Fig. 2 . In this scenario, we assume that both ends of the MIMO link are equipped with 4-element square arrays, [16, Fig. 1] and [17, Fig. 4]. This results in the idealized LOS matrix model

$$
\boldsymbol{H}_{0}:=\boldsymbol{H}_{0}^{(4)}=\mathrm{e}^{-j k D}\left(\begin{array}{cccc}
1 & a & a & b \\
a & 1 & b & a \\
a & b & 1 & a \\
b & a & a & 1
\end{array}\right)
$$

where

$$
\begin{aligned}
& a=\mathrm{e}^{-j k D\left(\sqrt{1+(d / D)^{2}}-1\right)} \approx \mathrm{e}^{-j \frac{k d^{2}}{2 D}} \\
& b=\mathrm{e}^{-j k D\left(\sqrt{1+2(d / D)^{2}}-1\right)} \approx \mathrm{e}^{-j k d^{2} / D}=a^{2} .
\end{aligned}
$$

\section{A. Eigen Decomposition}

After some tedious manipulations, it turns out that the eigenvalue $1-b$ has a multiplicity equal to two and consequently the eigendecomposition of $\boldsymbol{H}_{0}^{(4)}$ can be written as

$$
\begin{aligned}
& \boldsymbol{\Lambda}_{0}^{(4)}=\mathrm{e}^{-j k D}\left(\begin{array}{cccc}
1+2 a+b & 0 & 0 & 0 \\
0 & 1-b & 0 & 0 \\
0 & 0 & 1-b & 0 \\
0 & 0 & 0 & 1-2 a+b
\end{array}\right) \\
& \boldsymbol{V}_{0}^{(4)}=\boldsymbol{V}_{0} \otimes \boldsymbol{V}_{0} .
\end{aligned}
$$

We see that $2 \boldsymbol{V}_{0}^{(4)}$ is the Hadamard matrix of order 4. Thus, the eigenvectors of the $4 \times 4$ MIMO channel matrix can be computed from those of the $2 \times 2$ case by a Kronecker product. This relation is exact.

1) Kronecker product interpretation: The exact Kronecker product connection (16) between the two sets of eigenvectors can be extended to the channel matrices itself and the associated eigenvalues in an approximate sense as follows. If we accept the Taylor series approximation for the phase angles then $b \approx a^{2}$ in (14) and $\boldsymbol{H}_{0}$ as defined in (12) can also be interpreted as a Kronecker product

$$
\boldsymbol{H}_{0}^{(4)} \approx \mathrm{e}^{-j k D}\left(\begin{array}{cc}
1 & a \\
a & 1
\end{array}\right) \otimes\left(\begin{array}{cc}
1 & a \\
a & 1
\end{array}\right) .
$$

Similarly, (15) can be read as the Kronecker product

$$
\boldsymbol{\Lambda}_{0}^{(4)} \approx \mathrm{e}^{-j k D}\left(\begin{array}{cc}
1+a & 0 \\
0 & 1-a
\end{array}\right) \otimes\left(\begin{array}{cc}
1+a & 0 \\
0 & 1-a
\end{array}\right)
$$

\section{B. Singular Value Decomposition}

The SVD of $\boldsymbol{H}_{0}^{(4)}=\boldsymbol{U}_{0}^{(4)} \boldsymbol{S}_{0}^{(4)} \boldsymbol{V}_{0}^{(4) H}$ is essentially identical to the eigendecomposition, given in (15) and (16). In addition, we can exploit the Kronecker product,

$$
\begin{aligned}
\boldsymbol{U}_{0}^{(4)} & =\boldsymbol{U}_{0} \otimes \boldsymbol{U}_{0} \\
\boldsymbol{S}_{0}^{(4)} & =\operatorname{diag}(|1+2 a+b|,|1-b|,|1-b|,|1-2 a+b|) \\
\boldsymbol{V}_{0}^{(4)} & =\boldsymbol{V}_{0} \otimes \boldsymbol{V}_{0} .
\end{aligned}
$$

\section{Eigenbeam Transmission With}

\section{LATTICE-Reduction Aided Zero Forcing}

We closely follow the proposed scheme in [18, Sec. IV.B]. In particular, let the MIMO transceiver be modeled as

$$
\boldsymbol{y}(t)=\boldsymbol{H}(t) \boldsymbol{s}(t)+\boldsymbol{n}(t),
$$

where $\boldsymbol{y}(t)$ is the complex-valued received data vector, $s$ is the transmitted vector symbol, and $\boldsymbol{n}(t)$ is additive white Gaussian noise. We use the Lenstra-Lenstra-Lovász (LLL) algorithm to calculate the unimodular matrix $\Pi(t)$ such that

$$
\boldsymbol{y}(t)=\tilde{\boldsymbol{H}}(t) \boldsymbol{z}(t)+\boldsymbol{n}(t),
$$

where $\tilde{\boldsymbol{H}}(t)=\boldsymbol{H}(t) \boldsymbol{\Pi}(t)$ and $\boldsymbol{z}(t)=\boldsymbol{\Pi}^{-1}(t) \boldsymbol{s}(t)$. We set the LLL algorithm parameter $\delta=\frac{3}{4}$, cf. [18]. The matrix $\tilde{\boldsymbol{H}}(t)$ has a lower condition number than $\boldsymbol{H}(t)$ with a higher probability. We therefore implement a zero forcing equalizer for the transformed model (21) and carry out the slicing on $\boldsymbol{z}(t)$ instead of $\boldsymbol{s}(t)$. The resulting receiver is non-linear because the slicing is carried out in the transformed domain.

\section{Bit Error Ratio Simulation}

Simulations have been carried out to evaluate bit error ratios (BER) for $2 \times 2$ and $4 \times 4$ MIMO systems at the carrier frequency of $f=32 \mathrm{GHz}$ in dry weather conditions $(K \rightarrow \infty)$. The distance between transmitter and receiver is set equal to $D=5 \mathrm{~km}$. The simulation is carried out for uncoded 16-QAM symbols on both Tx antenna elements with Gray mapping. The simulated MIMO channel realizations are drawn randomly according to (1) for $d=2.4198 \mathrm{~m}, \varrho=0.1$, and the elements of $\boldsymbol{W}$ are independent random variables and identically distributed. These settings result in $a \approx \mathrm{e}^{-j \pi / 8}$. Please note that, following the methodology of [8], [9], selecting $d=4.8395 \mathrm{~m}$ would result in $a \approx \mathrm{e}^{-j \pi / 2}=-j$ and $\boldsymbol{H}_{0}$ would become unitary. This implies that the following simulations are carried out with an inter-element spacing that is approximately equal to half the optimal spacing.

Figure 3 shows the obtained BER for the $2 \times 2$ MIMO connection for three types of MIMO transceivers. The BER result for zero forcing based on (20) is labeled "perfect $\mathrm{Rx}$ ZF". The BER result for zero forcing based on (21) is labeled "LLL-aided perfect Rx ZF". We also show the BER result of 
the exact maximum likelihood receiver which carries out an exhaustive search over the whole Tx symbol vector alphabet. (labeled "perfect Spatial Muxing ML").

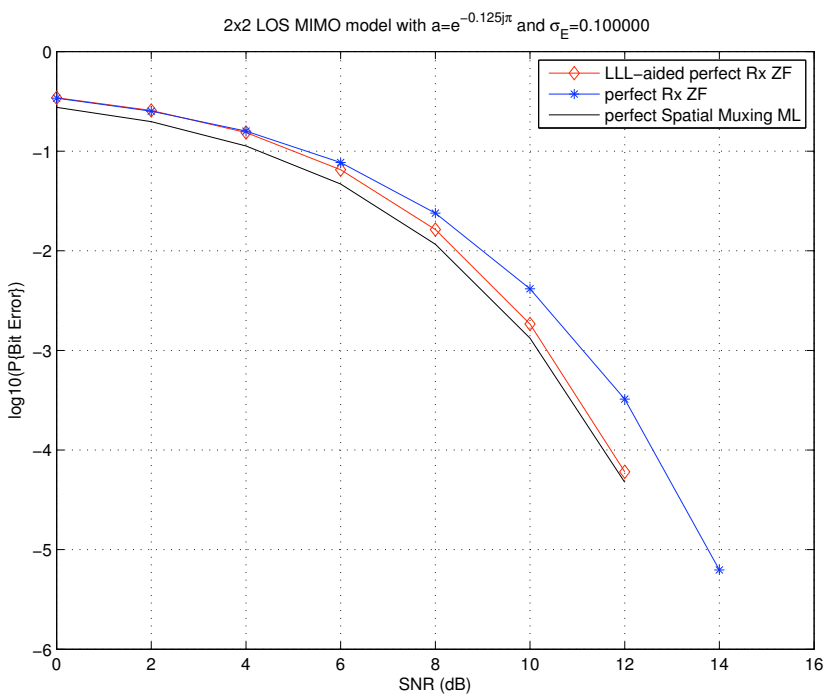

Fig. 3. $2 \times 2$ LOS MIMO, 16-QAM, Gray mapping, $a=\mathrm{e}^{-j \pi / 8}(D=5 \mathrm{~km}$, $d=2.4198 \mathrm{~m}, 32 \mathrm{GHz})$ and $\varrho=0.1$, elements of $\boldsymbol{H}_{\mathrm{GR}}$ are i.i.d.

We observe that the BER of all three types of receivers is very similar in behavior. The LLL-aided zero forcing scheme outperforms standard zero forcing and achieves close to maximum-likelihood performance.

Next, Fig. 4 shows the obtained BER for the $4 \times 4$ MIMO connection for the same three types of MIMO transceivers. The BER result for zero forcing based on (20) is labeled "perfect Rx ZF". The BER result for zero forcing based on (21) is labeled "LLL-aided perfect Rx ZF". We also show the BER result of the exact maximum likelihood receiver which carries out an exhaustive search over the whole Tx symbol vector alphabet. (labeled "perfect Spatial Muxing ML").

When comparing Figs. 3 and 4, we note that the BER of these three types of receivers differs more in the latter $(4 \times$ 4) than in the former $(2 \times 2)$. We note that the LLL-aided zero forcing scheme achieves close to maximum-likelihood performance although the random $4 \times 4$ MIMO channel matrix is far from unitary.

Finally, we investigate the gain for the $4 \times 4$ setting with a spatially correlated ground reflection. We simulate $\boldsymbol{H}_{\mathrm{GR}}$ according to (2). We assume $\varrho=0.1$ as before, $\boldsymbol{R}=\boldsymbol{T}$ and the elements of $\boldsymbol{R}$ are defined by (24) for $\beta=20^{\circ}, \vartheta_{0}=0^{\circ}$. The results are shown in Fig. 5.

\section{CONCLUSIONS}

The Tx eigenbeamforming vectors for fixed $2 \times 2$ LOS MIMO links can be defined without CSIT. The eigenbeamforming vectors are defined by the Hadamard matrix which effectively forms sum and difference beams. Further, we have shown how these results carry over to a $4 \times 4$ LOS MIMO configuration by exploiting an underlying Kronecker structure.

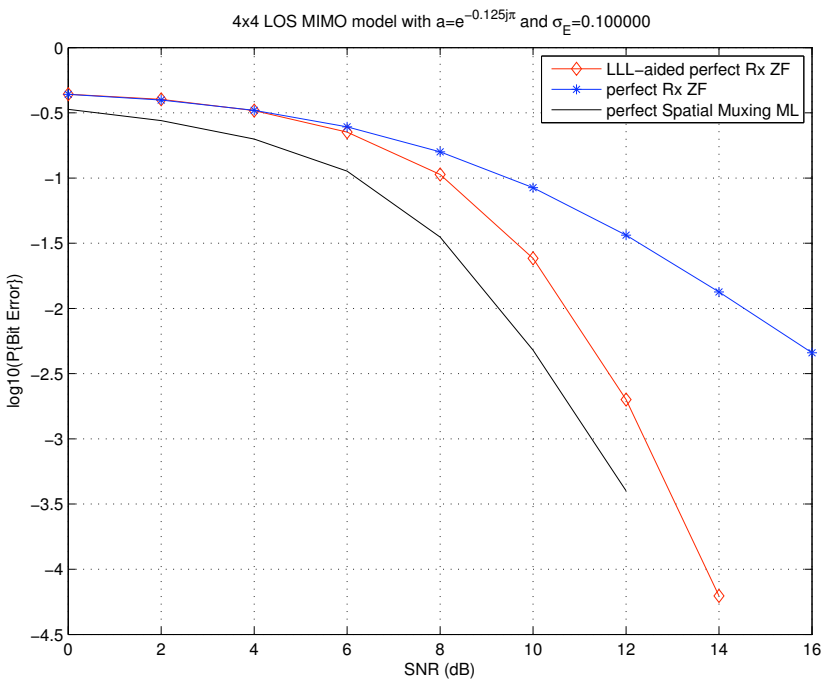

Fig. 4. $4 \times 4$ LOS MIMO, 16-QAM, Gray mapping, $a=\mathrm{e}^{-j \pi / 8}(D=5 \mathrm{~km}$, $d=2.4198 \mathrm{~m}, 32 \mathrm{GHz})$ and $\varrho=0.1$, elements of $\boldsymbol{H}_{\mathrm{GR}}$ are i.i.d.

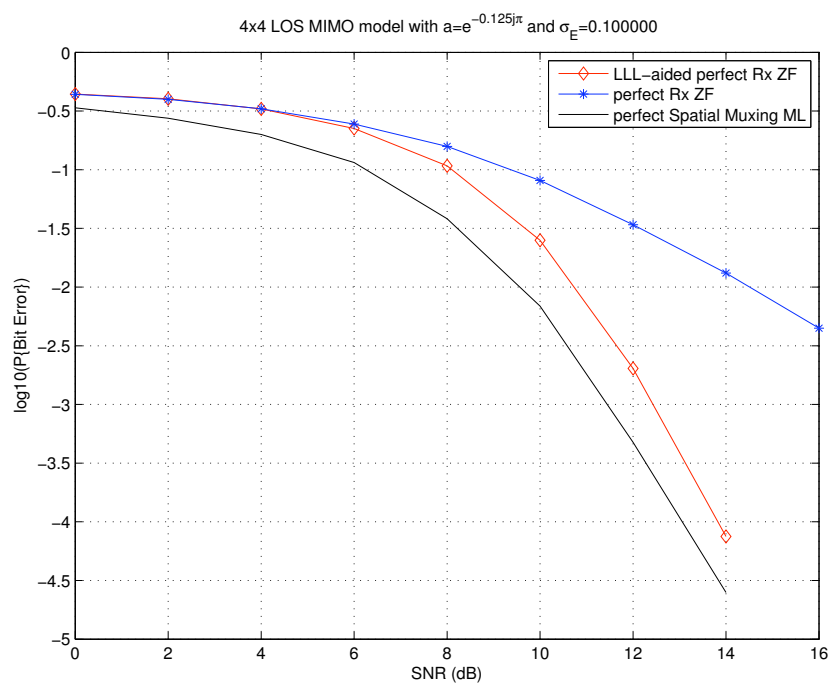

Fig. 5. $4 \times 4$ LOS MIMO with spatially correlated ground reflection, 16QAM, Gray mapping, $a=\mathrm{e}^{-j \pi / 8}(D=5 \mathrm{~km}, d=2.4198 \mathrm{~m}, 32 \mathrm{GHz})$ and $\varrho=0.1, \boldsymbol{H}_{\mathrm{GR}}$ according to (2) and (24) with $\beta=20^{\circ}$ and $\vartheta_{0}=0^{\circ}$.

We show that a convenient low-complexity transceiver can use eigenbeamforming at the transmitter while the baseband receiver signal processing implements lattice-reduction aided linear reception. By doing so, a near optimum performance can be achieved even for ill-conditioned LOS channel matrices. We investigated the corresponding transceivers by numerical simulations and conclude that the LLL-aided zero forcing scheme achieves close to maximum-likelihood performance.

\section{ACKNOWLEDGEMENTS}

We thank T. McKelvey and E. Ström for valuable comments, and D. Wübben for providing the Matlab implementation of the LLL algorithm. Christoph's visit at Chalmers University of Technology was supported by a stipend from Ericsson AB. 
This work was partially funded by the Christian Doppler Laboratory for Wireless Technologies for Sustainable Mobility. The financial support by the Federal Ministry of Economy, Family and Youth and the National Foundation for Research, Technology and Development is gratefully acknowledged.

\section{APPENDIX}

This derivation closely follows [15, Appendix]. It is assumed that the ground reflection geometry is planar, i.e., the spread in azimuth shall be negligible compared to the spread in elevation $\vartheta$. Further, the antenna array is assumed to be a vertical linear array. In this case, the $(n, m)$-th element of the ground reflection covariance matrix $\boldsymbol{R}$ in (2) is given by [19]

$$
R_{n m}=\int_{0}^{2 \pi} w(\vartheta) \exp \left[j \frac{\omega}{c_{0}} d_{n m} \cos \left(\vartheta-\xi_{n m}\right)\right] \mathrm{d} \vartheta,
$$

where $d_{n m}$ is the distance between the antenna array elements $n$ and $m$. Further, $\xi_{n m}$ defines the orientation of the line which joins them. If the $n$-th array element is mounted higher above the ground than the $m$ th array element then $\xi_{n m}=0$ else $\xi_{n m}=\pi$.

The weighting function $w(\vartheta)$ describes the power distribution in elevation. Thus, $w(\vartheta) \geq 0$ for all $0 \leq \vartheta<\pi$ and $w(\vartheta)$ can be assumed to be periodic with period $2 \pi$. Now, let $w(\vartheta)$ be expandable in a Fourier series

$$
w(\vartheta)=\sum_{\ell=-\infty}^{\infty} w_{\ell} \mathrm{e}^{-j \ell \vartheta}, \text { with } w_{\ell}=\frac{1}{2 \pi} \int_{-\pi}^{\pi} w(\vartheta) \mathrm{e}^{j \ell \vartheta} \mathrm{d} \vartheta .
$$

The Fourier series is inserted into (22) resulting in

$$
\begin{aligned}
& R_{n m}= \\
& \quad \sum_{\ell=-\infty}^{\infty} w_{\ell} \int_{-\pi}^{\pi} \exp \left(j \frac{\omega}{c_{0}} d_{n m} \cos \left(\vartheta-\xi_{n m}\right)-j \ell \vartheta\right) \mathrm{d} \vartheta .
\end{aligned}
$$

The remaining integral is recognized as $2 \pi \exp \left(j \xi_{n m}\right)=$ $\pm 2 \pi$-times the Bessel function $\mathrm{J}_{\ell}(\cdot)$ of the first kind and order $\ell$, yielding

$$
R_{n m}=2 \pi \sum_{\ell=-\infty}^{\infty} w_{\ell} \mathrm{e}^{j \ell \xi_{n m}} \mathrm{~J}_{\ell}\left(\frac{\omega}{c_{0}} d_{n m}\right) .
$$

An idealized ground reflection with elevation spread $2 \beta$ is e.g. described by the rectangular power weighting function $w(\vartheta)=0$ for $\left|\vartheta-\vartheta_{0}\right|>\beta$ and $w(\vartheta)=1$ for $\left|\vartheta-\vartheta_{0}\right|<\beta$. In this case, the Fourier coefficients become

$$
w_{\ell}= \begin{cases}\frac{\beta}{\pi}, & \text { for } \ell=0 \\ \frac{\sin (\beta \ell)}{\pi \ell}, & \text { else }\end{cases}
$$

\section{REFERENCES}

[1] F. Bøhagen, P. Orten, G. E. Øien, and S. de la Kethulle de Ryhove, "Exact capacity expressions for dual-branch Rician MIMO channels," IEEE Trans. Commun., vol. 56, no. 12, pp. 2214-2222, Dec. 2008.

[2] S. Jin, M. R. McKay, X. Gao, and I. B. Collings, "MIMO multichannel beamforming: SER and outage using new eigenvalue distributions of complex noncentral Wishart matrices," IEEE Trans. Commun., vol. 56, no. 3, pp. 424-434, Mar. 2008.
[3] M. Matthaiou, P. de Kerret, G. K. Karagiannidis, and J. A. Nossek, "Mutual information statistics and beamforming performance analysis of optimized LoS MIMO systems," IEEE Trans. Commun., vol. 58, no. 11, pp. 3316-3329, Nov. 2010.

[4] A. M. Tulino, A. Lozano, and S. Verdú, "Capacity-achieving input covariance for single-user multi-antenna channels," IEEE Trans. Wireless Commun., vol. 5, no. 3, pp. 662-671, Mar. 2006. [Online]. Available: http://www.princeton.edu/ verdu/reprints/ALATSVmar2006.pdf

[5] E. Riegler and G. Taricco, "Second-order statistics of the mutual information of the asymptotic separately-correlated Rician fading MIMO channel with interference," in Proc. IEEE GLOBECOM, Washington, DC, USA, November 26-30 2007.

[6] _ "On the ergodic capacity region of the separately correlated Rician fading multiple access MIMO channel," in Proc. IEEE GLOBECOM, Washington, DC, USA, November 26-30 2007.

[7] R. U. Nabar, H. Bölcskei, and A. J. Paulraj, "Diversity and outage performance in space-time block coded Ricean MIMO channels," IEEE Trans. Wireless Commun., vol. 4, no. 5, pp. 2519-2532, September 2005.

[8] F. Bøhagen, P. Orten, G. E. Øien, and S. de la Kethulle de Ryhove, "Construction and capacity analysis of high-rank line-of-sight mimo channels," in Proc. IEEE Wireless Commun. Networking Conf. (WCNC), New Orleans, LA, Mar. 2005, pp. 432-437.

[9] I. Sarris and A. R. Nix, "Design and performance assessment of high-capacity mimo architectures in the presence of a line-of-sight component," IEEE Trans. Veh. Technol., vol. 56, no. 4, pp. 2194-2202, Jul. 2007.

[10] F. Bøhagen, P. Orten, and G. E. Øien, "Modeling and analysis of a $40 \mathrm{GHz}$ MIMO system for fixed wireless access," in Proc. IEEE Veh. Technol. Conf. 2005 spring, Stockholm, Sweden, May 2005.

[11] F. Bøhagen, "Modeling, characterization and design of line-of-sight wireless MIMO channels," Ph.D. dissertation, University of Oslo, Oslo, Norway, February 2007.

[12] T. Ingason and L. Haonan, "Line-of-sight MIMO for microwave links," Master's thesis, Chalmers University of Technology, Gothenburg, Sweden, 2009.

[13] T. Ingason, H. Liu, M. Coldrey, A. Wolfgang, and J. Hansryd, "Impact of frequency selective channels on a line-of-sight MIMO microwave radio link," in Proc. IEEE Veh. Technol. Conf. 2010 spring, Taipei, Taiwan, 16-19 May 2010.

[14] H. Xu, T. S. Rappaport, R. J. Boyle, and J. H. Schaffner, "Measurements and models for $38-\mathrm{GHz}$ point-to-multipoint radiowave propagation," IEEE J. Sel. Areas Commun., vol. 18, no. 3, pp. 310-321, Apr. 2000.

[15] M. Haardt, C. F. Mecklenbräuker, M. Vollmer, and P. Slanina, "Smart antennas for UTRA TDD," European Transactions on Telecommunications, vol. 12, no. 5, pp. 393-406, Sep. 2001.

[16] L. Liu, M. Wei Hong, H. Wang, G. Yang, N. Zhang, H. Zhao, J. Chang, C. Yu, X. Yu, H. Tang, H. Zhu, and L. Tian, "Characterization of line-of-sight MIMO channel for fixed wireless communications," IEEE Antennas Wireless Propag. Lett., vol. 6, pp. 36-39, 2007.

[17] F. Bøhagen, P. Orten, and G. Øien, "Optimal design of uniform rectangular antenna arrays for strong line-of-sight MIMO channels," EURASIP Journal on Wireless Communications and Networking, p. 10, 2007.

[18] D. Wübben, R. Böhnke, V. Kühn, and K.-D. Kammeyer, "Nearmaximum-likelihood detection of MIMO systems using MMSE-based lattice-reduction," in Proc. IEEE Int. Conf. Commun., vol. 2, Paris, France, June 2004, pp. 798-802.

[19] B. F. Cron and C. H. Sherman, "Spatial-correlation functions for various noise models," Journal of the Acoustical Society of America, vol. 34, no. 11, pp. 1732-1736, Nov. 1962. 